Research Article

\title{
Investigation of Microwave Irradiation Procedure for Synthesizing CdSe Quantum Dots
}

\author{
Jacob Strimaitis $\mathbb{D}^{1},{ }^{1}$ Taliya Gunawansa, ${ }^{1}$ Sangram Pradhan, ${ }^{1}$ and Messaoud Bahoura ${ }^{1,2}$ \\ ${ }^{1}$ Norfolk State University, Center for Materials Research, 555 Park Ave, Norfolk, VA 23504, USA \\ ${ }^{2}$ Norfolk State University, Engineering Department, 555 Park Ave, Norfolk, VA 23504, USA \\ Correspondence should be addressed to Jacob Strimaitis; j.strimaitis@spartans.nsu.edu
}

Received 17 October 2019; Revised 11 January 2020; Accepted 20 January 2020; Published 29 February 2020

Academic Editor: Francesco Ruffino

Copyright (C) 2020 Jacob Strimaitis et al. This is an open access article distributed under the Creative Commons Attribution License, which permits unrestricted use, distribution, and reproduction in any medium, provided the original work is properly cited.

\begin{abstract}
In recent years, microwave heating techniques for quantum dot (QD) synthesis have come to supplement the typical hot-injection methods. In addition to increasing control and replicability, microwave synthesis can be up-scaled to industry standards, an advantage that increases its lucrativeness. This study depicts a strategy to take a hot-injection procedure for cadmium selenide (CdSe) QD synthesis that is safe enough for undergraduate research labs and adapt it to an easier, more energy-efficient microwave synthesis method. Additionally, this study details successes in synthesizing these QDs, along with some challenges, limitations, and peculiarities. For future users of this method, it is recommended to keep holding temperatures between $170^{\circ} \mathrm{C}$ and $240^{\circ} \mathrm{C}$ to achieve the highest monodispersity of CdSe QDs while also avoiding confounding effects, such as wide-spectrum photoluminescence and bulk CdSe precipitation.
\end{abstract}

\section{Introduction}

Quantum dots (QDs) are semiconducting nanoparticles with intriguing optoelectronic properties brought about by the quantum confinement effect [1-3]. Specifically, as the size of the QDs approaches the nanoscale, they gain the ability to absorb and emit higher-energy radiation than their bulk material counterparts [3]. These unique phenomena have been the impetus behind the inclusion of QDs into thin films serving the photovoltaic [4, 5], light-emitting diode [6], and other energy utilization industries [7]. Despite being relatively easy to synthesize with basic laboratory equipment found in most undergraduate organic or physical chemistry labs [8], QDs are still emerging materials that require more research to increase their processing viability, and by extension, integration in future devices.

The most widely used method for QD synthesis is the hot-injection approach [8-12]. This method involves two essential components: a hot reaction flask and an injection precursor. In the reaction flask, a solvent with a high boiling point is heated, usually to $\sim 200^{\circ} \mathrm{C}$ or higher. In a separate area, precursors with the components of the QDs are heated or stirred until they are completely dissolved. Then, the precursors are quickly injected into the hot reaction flask, causing an immediate nucleation of QDs via supersaturation. Over time, as the cooled reaction flask heats back up, the nanocrystals grow. At pointed intervals throughout the growth process, aliquots of the reaction flask are taken, with smaller nanoparticles taken early on and larger nanoparticles taken later. These acquired QDs are in colloidal form (i.e., suspended, but neither dissociated nor precipitated in the solvent) and can be purified, characterized, or otherwise manipulated to suit the needs of the researcher.

The microwave irradiation technique, by contrast, is a newer approach designed to reduce some of the frustrations and limitations of the older hot-injection method. To list a few, the hot-injection method suffers from a lack of control, reproducibility, and large-scale synthesis, whereas the microwave synthesis technique excels on all three counts [13]. Regarding control and reproducibility, the effects of ambient air conditions and uneven heating within the reaction flask make the hot-injection method inferior to the microwave. 
The microwave method works within a sealed environment under temperature ranges that can be precisely reproduced. Furthermore, the hot-injection method cannot reach the same level of homogeneity in large-scale operations as it can in small-scale environments; conversely, microwave syntheses allow for homogeneity as all the components can be prepared separately and allowed to settle in a mixed batch prior to heating.

Owing to its usefulness, there have been a variety of successful attempts to make many types of QDs via the microwave synthesis method [13-20]. While the merits and scientific processes of the microwave approach have already been described in these attempts, lesser attention has been given to some of the approach's setbacks. The two main goals of this investigation are to provide an option for microwave synthesis that originates from a commonplace hot-injection method and, more importantly, highlight some of the challenges, limitations, and peculiarities that are not normally reported in other microwave synthesis studies $[8,12,18,19]$. Such a problem-centered approach might benefit the researcher, student, or industry professional exploring the phenomenological limits of QDs.

\section{Method}

2.1. List of Chemicals. Selenium powder (Se, 99.999\%, Alfa Aesar), tri-n-octylphosphine (TOP, min. 97\%, Strem Chemicals, Inc.), cadmium acetate hydrate $\left(\mathrm{CdAc}_{2} \cdot x \mathrm{H}_{2} \mathrm{O}\right.$, $\geq 99.99 \%$, Sigma-Aldrich), oleic acid (OA, tech. 90\%, Alfa Aesar), 1-octadecene (ODE, tech. 90\%, Alfa Aesar), toluene (ACS reagent, 99.5\%, Alfa Aesar), acetone (ACS reagent, min. 99.5\%, VWR), chloroform (min. 99.8\%, BDH-VWR), and hexane ( $n$-hexane, $\min$. 60\%; C6 isomers, $\min .98 .5 \%$; BDH-VWR).

2.2. Precursor Preparations. As per the instructions of the method on which this study is based [8], the Se precursor was prepared by dissolving $99 \mathrm{mg}$ of Se powder in $5.5 \mathrm{~mL}$ of TOP. Though easier to do in a round-bottom flask where the sediments can be continually agitated with the stirring element, we were able to dissolve the Se powder in $20 \mathrm{~mL}$ glass vials by moving the vial (and accompanying stir bar) across the stir plate to break up some of the bulkier chunks. At room temperature, complete dissolution took 1-2 hours. After even a few hours of exposure to air, Se precursors would show spots of crystallization on the interior of the glass. However, this decomposition was likely the oxidation of TOP into tri-n-octylphosphine oxide (TOPO), which is a common native ligand in QD chemistry and thus not cause for immediate concern [21].

The Cd precursor was prepared by dissolving $53 \mathrm{mg}$ of $\mathrm{CdAc}_{2} \cdot x \mathrm{H}_{2} \mathrm{O}$ in a $20 \mathrm{~mL}$ glass vial containing $0.6 \mathrm{~mL}$ of OA and $5.5 \mathrm{~mL}$ of ODE. Complete dissolution occurred after stirring and heating to $130^{\circ} \mathrm{C}$, which usually took two hours. Solutions were cooled back down to room temperature before being implemented for synthesis. Though the Cd precursor did not degrade as quickly on a laboratory tabletop as the Se precursor, it would still show signs of degradation in the form of a wispy precipitate after 1-2 days.

All of the QD batches for this report were made within 12 days of preparing their precursors, and if the precursors were not clear and colorless on the day of synthesis, even after reheating, they were discarded and prepared again. Precursors made with these procedures can make five batches of QDs, with room to scale up as needed [8].

2.3. Microwave Synthesis. The procedure for microwave synthesis was the same as that of the hot-injection method in a previous report [8], save for the inclusion of a microwave (Anton Paar Monowave 300) as the primary heating element and the distinction that precursors were mixed into the reaction vial (size G30) at room temperature instead of near $200^{\circ} \mathrm{C}$. Aliquots of the Se precursor $(1 \mathrm{~mL})$ and $\mathrm{Cd}$ precursor $(1 \mathrm{~mL})$ were added to the $10 \mathrm{~mL}$ of ODE already in the reaction vial, causing turbidity in the reaction solution without color change or precipitation. The reaction vial was then sealed with a snap cap and silicone septum, into which a glass immersion tube was inserted. A ruby thermometer, which was used to more accurately measure the increasing temperature of the reaction vial than the ambient temperature reading of built-in IR sensors [22], was then inserted into the immersion tube. The reaction vial, along with the ruby thermometer and a magnetic stir bar, was placed in the reaction cavity for synthesis.

Microwave reactors work in three steps: heating up, holding, and cooling down. During the heating up step, reactors provide maximum inputted power to heat up the sample to the desired temperature. For all the reactions in this study, the option to heat as fast as possible was selected, meaning that it took anywhere from 2 to 5 minutes to reach the inputted temperature. The maximum power was set at $300 \mathrm{~W}$, and stirring speed of the magnetic stir bar was set at $600 \mathrm{rpm}$. During the holding step, reactors fluctuate power levels to maintain a desired temperature. The holding temperature for each experiment in this study was set between $140^{\circ} \mathrm{C}$ and $280^{\circ} \mathrm{C}$, with hold times between 0 and 5 minutes. These important holding parameters are consistent with other similar QD microwave synthesis procedures $[18,20]$. During the cooling down step, reactors blow cool air into the reaction cavity and expel warm air through an exhaust pipe, a process which takes anywhere from 2 to 5 minutes to reach the desired temperature. The target cool down temperature for each experiment was $70^{\circ} \mathrm{C}$, at which point all stirring and cooling processes stopped and the reaction vial could be removed.

To illustrate, if one wished to reproduce a batch of CdSe QDs heated to $200^{\circ} \mathrm{C}$ for 3 minutes using our method, one would input the following parameters for the three aforementioned steps: for the heating up step, one would select "heat as fast as possible," $300 \mathrm{~W}$ for the maximum power, and $600 \mathrm{rpm}$ for the magnetic stir bar speed. For the holding step, one would select $200^{\circ} \mathrm{C}$ for holding temperature, 3 minutes for hold time, and $600 \mathrm{rpm}$ again for the magnetic stir bar speed. For the final cooling step, one would select $70^{\circ} \mathrm{C}$ as the target temperature and $600 \mathrm{rpm}$ again for the 
magnetic stir bar speed. After inserting the reaction vial and ruby thermometer, one would simply tap the start button to begin the procedure, letting the microwave heat up, hold, and cool down according to the set parameters. The solution in the reaction vial at the end would contain the colloidal QDs ready for purification or analysis.

2.4. Cleaning. Previous findings suggest that cleaning QDs is a viable way to remove unreacted impurities from QD solutions [23]. Based on a method from another report [8], we attempted to clean the QDs by inserting $2 \mathrm{~mL}$ of QD solution, $0.6 \mathrm{~mL}$ of OA, and either $2.6 \mathrm{~mL}$ or $5.2 \mathrm{~mL}$ of acetone into a $\sim 30 \mathrm{~mL}$ centrifuge tube, which was then spun at $4,000 \mathrm{rpm}$ for $10 \mathrm{~min}$. This procedure with either amount of acetone, however, yielded no QD pellets. After some trialand-error, it was determined that between 1 and $2 \mathrm{~mL}$ of QD solution (though we used $1.4 \mathrm{~mL}$ for all experiments after this one) in a tube with $28 \mathrm{~mL}$ of acetone spinning at $10,000 \mathrm{rpm}$ for 15 minutes was enough to get pellets between 1 and $5 \mathrm{~mm}$ in diameter. These pellets also ranged in color from pale yellow to red-orange, depending on the QDs used.

After precipitation in the polar solvent via centrifugation, supernatants containing excess impurities were discarded. Pellets were then resuspended in $1.25 \mathrm{~mL}$ of the target solvent, which was either ODE, toluene, hexane, or chloroform [24]. Save for unusual exceptions noted in Section 3, resuspensions were transparent and monochromatic between yellow and red-orange under visible light.

\section{Results and Discussion}

3.1. PL-Broadening, UV-Vis, and Size. As seen in Figure 1(a), solutions of QDs immediately after microwave synthesis range in color from pale yellow to dark red under visible light. These colors are consistent with those from other reports with hot-injection procedures $[8,12]$. Most QD syntheses are also transparent, though some batches near the upper limit of the microwave reactor appear cloudy with a dark red precipitate (presumably bulk CdSe powder). Batches made below $120^{\circ} \mathrm{C}$ show no color change or luminescence under UV light, suggesting that there is a lower limit of temperature beyond which QD nucleation does not occur.

QDs synthesized within the working range of temperatures show odd patterns of emission under $365 \mathrm{~nm}$ UV light (Figure 1(b)). Instead of fluorescing the usual blue to red $[8,12]$, QDs emit colors ranging from a silver-blue, to bright white, to a peachy orange. These abnormalities appear in the PL spectra as well (Figure 2(b)), where broad-spectrum emissions dominate for lower temperature synthesis batches (long-lined curves). Batches made between $170^{\circ} \mathrm{C}$ and $240^{\circ} \mathrm{C}$ have more defined peaks consistent with other reports $[8,12]$, yet even these QDs show inexplicable broad emission in the range of $650-750 \mathrm{~nm}$.

With UV-vis spectroscopy, however, the QDs display a more common pattern (Figure 2(a)). Absorption peaks fall between 430 and $580 \mathrm{~nm}$, a range that lies within the visible light spectrum between green and yellow. A representative batch of QDs also shows the familiar Stokes shift (Figure 3) $[8,12,18,19,25]$, indicating the difference in energy between absorbed photons and those that are emitted after nonradiative relaxation.

Transmission electron microscope (TEM) images of QDs synthesized in our lab have been published in a previous report [26]. In colloidal form, QDs are relatively spherical in shape with an inner core composed of lattice CdSe and an outer shell composed of attached organic ligands [8]. The size of the QDs can be approximated using photoluminescence peak data and the following Brus equation $[1,2]$ :

$$
R^{2}=\frac{h^{2}}{8 E_{\mathrm{x}}}\left(\frac{1}{m_{e}^{*}}+\frac{1}{m_{h}^{*}}\right),
$$

where $E_{x}=E-E_{\mathrm{g}}(\mathrm{CdSe}), E_{\mathrm{g}}(\mathrm{CdSe})=1.74 \mathrm{eV}, m_{e}^{*}(\mathrm{CdSe})$ $=0.13 m_{\mathrm{o}}\left(m_{\mathrm{o}}\right.$ is the mass of free electron), $m_{h}^{*}$ (CdSe) $=0.45 \mathrm{~m}_{\mathrm{o}}$, and $E$ is calculated using the de Broglie relation, $E=h c / \lambda$, where $h$ is Planck's constant and $\lambda$ is the peak PL wavelength of the QDs. Table 1 depicts the results of these calculations.

3.2. Blue Shift from Cleaning. One exciting feature of QDs is that they appear to blue shift after cleaning. This phenomenon is represented in Figure 4, where QDs synthesized at various hold temperatures appear one color just after microwave synthesis but show another color (specifically blue-shifted) after cleaning in acetone and resuspension in toluene. This effect is not limited to visible light; under a lowpowered UV lamp (365 nm), QDs also fluoresce a bluer color than their unpurified counterparts.

Other groups have cleaned QDs without reporting a blue shift $[8,23]$. The removal of impurities is supposed to increase the signal-to-noise ratio of PL spectra, specifically with the removal of minor peaks [8], and have little effect on the PL peak location. The fact that our QDs blue-shifted so dramatically, by contrast, suggests that something unintended is happening during cleaning. One explanation for this phenomenon is the removal of neutral, coordinating ligands. It is unlikely that the ligands are replaced entirely by either acetone or toluene, but there is evidence that suggests some ligands, such as TOP, are removed during the cleaning process [23]. The decrease in surfactant attachments may decrease the overall size of the QDs to the point that they respond to photonic excitation like smaller nanocrystals. Another possible explanation is the fragmentation of aggregate QDs during the high-speed cleaning process. The replacement of aggregate QDs (red light) with a larger amount of smaller QDs (blue light) would increase the overall intensity of bluer light.

3.3. Resuspension Solvent Choice. QDs are covered in longchained ligands to disallow aggregation and enable easy solution processing $[8,11,27]$. According to the principle "like dissolves like," the closer the shape and polarity a molecule or surfactant is to its solvent, the more likely it is to 


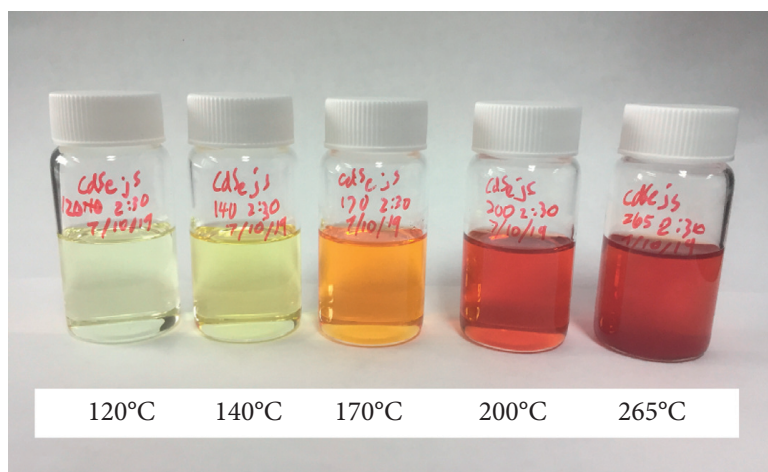

(a)

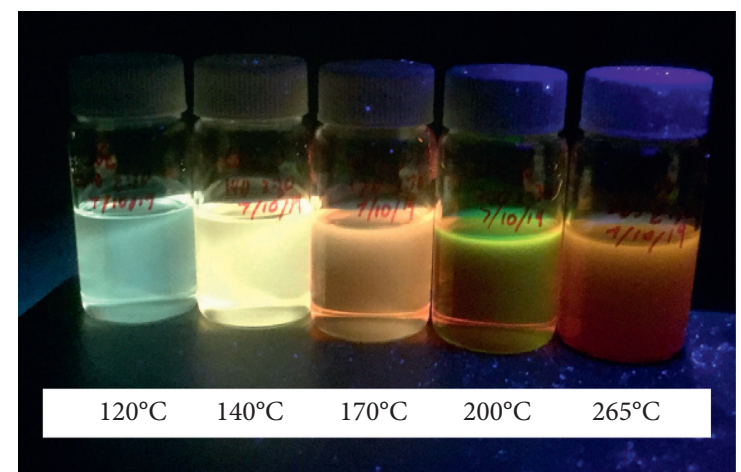

(b)

FIGURE 1: Array of quantum dots in glass vials immediately after microwave synthesis under visible light (a) and under $365 \mathrm{~nm}$ UV light (b). From left to right, hold temperatures were $120^{\circ} \mathrm{C}, 140^{\circ} \mathrm{C}, 170^{\circ} \mathrm{C}, 200^{\circ} \mathrm{C}$, and $265^{\circ} \mathrm{C}$, with hold time remaining constant at $2 \mathrm{~min} 30 \mathrm{~s}$.

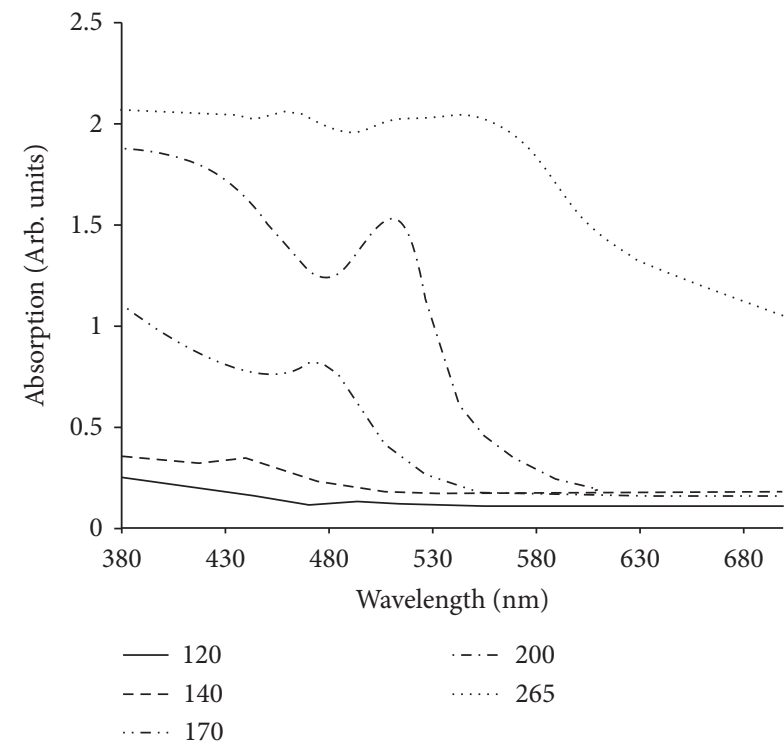

(a)

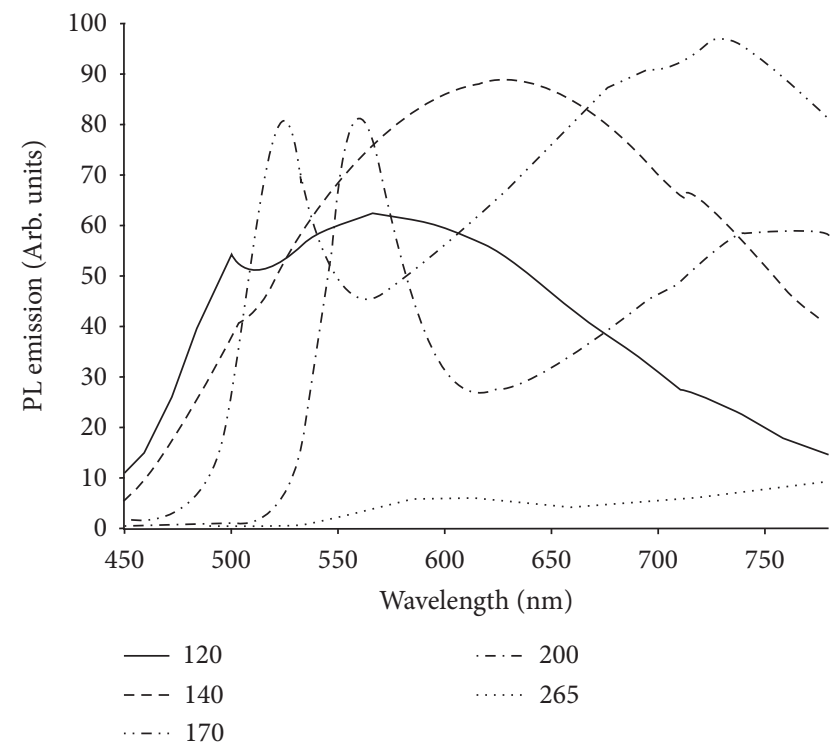

(b)

Figure 2: Comparison of UV-vis absorption (a) and PL emission $\left(\lambda_{\mathrm{ex}}=400 \mathrm{~nm}\right)$ (b) of QDs synthesized via the microwave method.

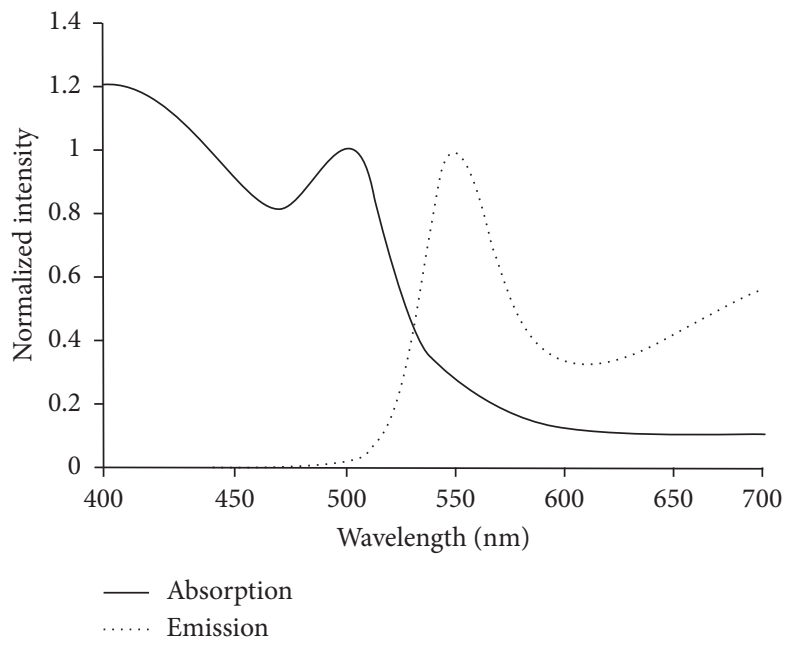

FIgURE 3: Example of Stokes shift of QDs $\left(200^{\circ} \mathrm{C}\right.$; hold time, 2 min $\left.30 \mathrm{~s}\right)$, showing that absorption (solid line) occurs at a higher energy than emission (dotted line) via photoexcitation at $400 \mathrm{~nm}$. 
TABLE 1: Comparison of microwave parameters for synthesis of CdSe QDs and the calculated sizes of those QDs based on estimated peak PL wavelengths.

\begin{tabular}{lcccc}
\hline Temperature $\left({ }^{\circ} \mathrm{C}\right)$ & Hold time $(\mathrm{min})$ & PL wavelength $(\mathrm{nm})$ & Calculated radius $(\mathrm{nm})$ & Calculated diameter $(\mathrm{nm})$ \\
\hline 120 & $2: 30$ & 575 & 3.0 & 6.0 \\
140 & $2: 30$ & 624 & 3.9 & 7.8 \\
170 & $2: 30$ & 523 & 2.4 & 4.8 \\
200 & $2: 30$ & 560 & 2.8 & 5.6 \\
265 & $2: 30$ & 605 & 3.5 & 7.0 \\
\hline
\end{tabular}

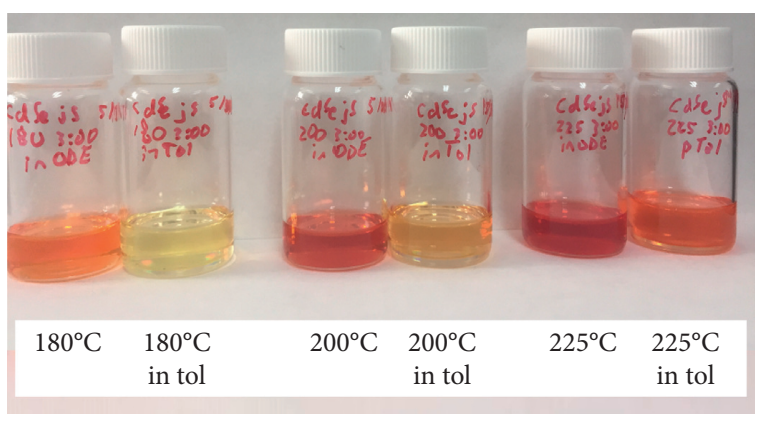

(a)

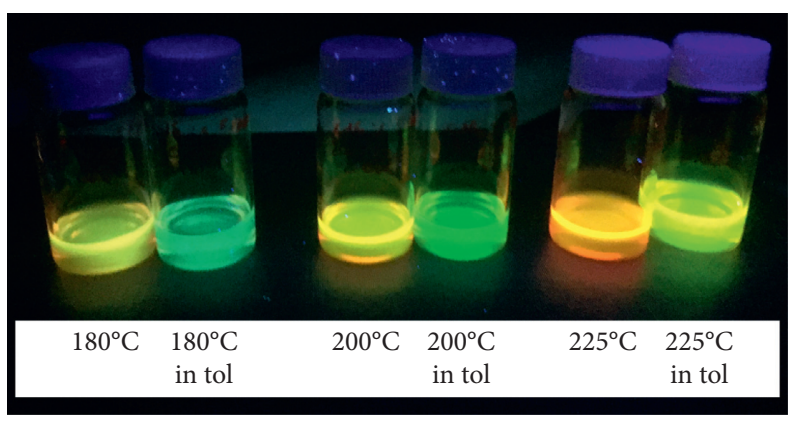

(b)

FIGURE 4: Images of QDs under visible light (a) and under $365 \mathrm{~nm}$ UV light (b) just before cleaning (left in each pair) and after resuspension in toluene (right in each pair). The batches were made with a hold time of $3 \mathrm{~min}$ and represent the following temperatures: $180^{\circ} \mathrm{C}$ (left pair), $200^{\circ} \mathrm{C}$ (middle pair), and $225^{\circ} \mathrm{C}$ (right pair).

dissolve in said solvent. This reasoning is what fuels the common assumption that QDs can be dissolved in most nonpolar organic solvents [24] since the long-chained ligands of QDs are themselves nonpolar. Surprisingly, this principle does not apply in full to QDs synthesized via this microwave method. In fact, resuspension only works well with toluene and marginally well with 1-octadecene (Figure 5), whereas resuspension is unsuccessful with nonpolar hexane and nonpolar chloroform. Even if some coloration occurs in solution, as is the case for resuspended CdSe QDs in hexane, they still will not fluoresce under UV light.

3.4. Photobrightening and Photobleaching. Ambient light can play a role in altering the stability of QDs in air, exemplified with two phenomena known as photobrightening and photobleaching [6]. Photobrightening is the tendency for ensembles of QDs to show increasing photoluminescence behavior under continuous photoexcitation [28], whereas photobleaching is the tendency for QD lattice structures to permanently degrade under prolonged light exposure and therefore lose all photoluminescence behavior [29]. The QDs synthesized in this study showed evidence of both effects.

A day after synthesis, QDs from one batch $\left(170^{\circ} \mathrm{C}\right.$; $2 \mathrm{~min} 30 \mathrm{~s}$ ) were dispensed into three plastic spectrophotometer cuvettes, wrapped in Parafilm, and then placed either in a dark laboratory drawer, on top of a table $\sim 1.5 \mathrm{~m}$ away from an external window (double-paned, low-e tempered glass), or on top of a windowsill $\sim 3 \mathrm{~cm}$ away from the same external window. These positions exemplify locations where researchers might properly (in drawer) or improperly (on tabletop or windowsill) store samples after daily use. Light for this experiment came from two primary sources: the fluorescent bulbs on the ceiling and the natural sunlight from the window. The fluorescent bulbs were $32 \mathrm{~W}$, T8 bulbs with a color temperature of $6,500 \mathrm{~K}$, illuminating at an average of 2,565 lumens. There were 24 bulbs in total, separated into groups of three and distributed across the ceiling of the open lab room into eight light fixtures with crosshatched aluminum light diffusers. The approximate distance from the QD samples and a point normal to the ceiling was $1.75 \mathrm{~m}$. The lights remained on, day and night, throughout the course of the experiment. The sunlight coming from the window changed by day, but an eight-year report from the National Renewable Energy Laboratory indicates that the direct normal solar irradiance on Norfolk, VA (USA), varies between 4.6 and $5.0 \mathrm{kWh} / \mathrm{m}^{2} /$ day [30].

The QDs initially displayed little difference in photoluminescence (PL) in terms of intensity, shape, or location of peak emission wavelength, as expected with identical samples, but over the course of eight days, a few patterns emerged (Figure 6). The first is that all samples had an initial increase in PL over the first couple of days (even the drawer sample, which was exposed to light for short periods between PL testing), indicating the photobrightening effect (Figure 6(a)). The second is that, over the course of the rest of the experiment, both samples continually exposed to light eventually experienced a decrease in PL intensity from the stable $\sim 1.5 \mathrm{PL} / \mathrm{PL}_{0}$, with the sample on the windowsill dropping in intensity to the level of background noise by day 7 (Figure 6(a)). This evidence indicates that photobleaching from the sun and commercial fluorescent bulbs begins after a 


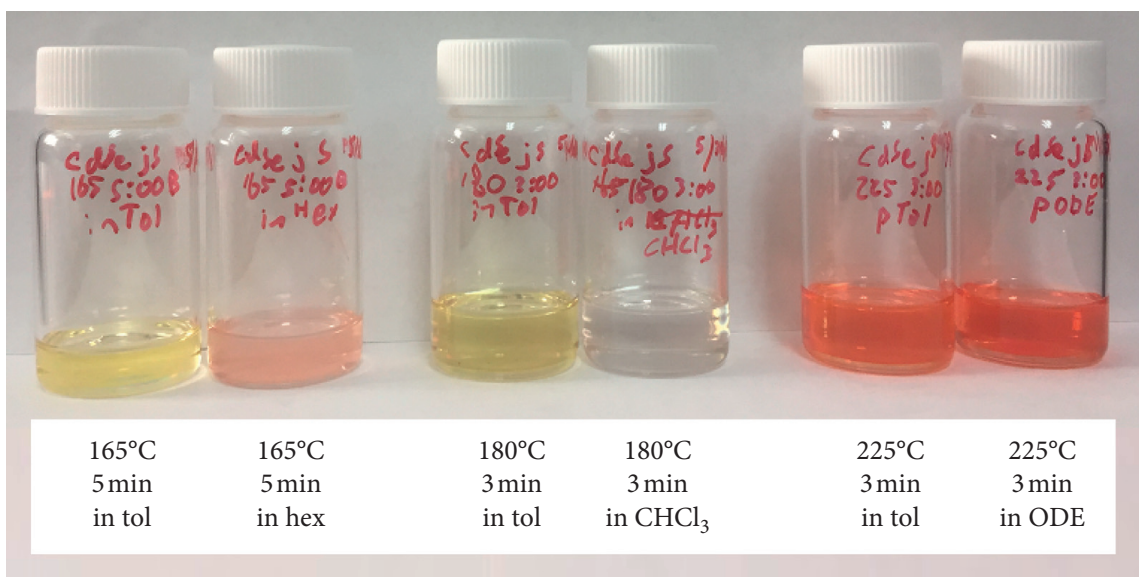

(a)

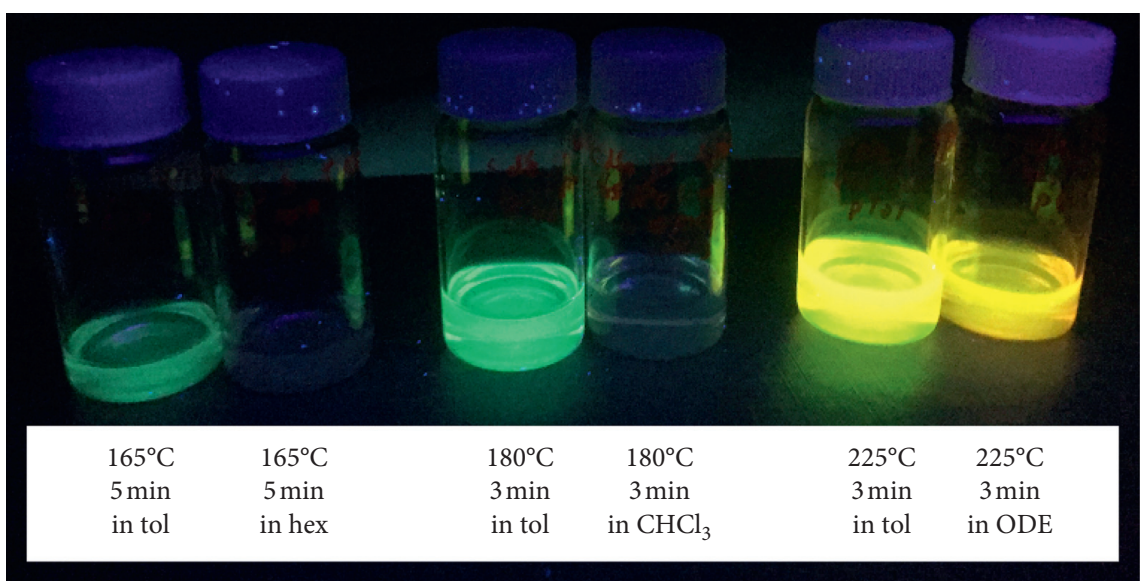

(b)

FIGURE 5: Comparison of QDs resuspended in different solvents under visible light (a) and $365 \mathrm{~nm}$ UV light (b). The left pair compares QDs prepared at $165^{\circ} \mathrm{C}$ for $5 \mathrm{~min}$ and resuspended in either toluene (left) or hexane (right). The middle pair compares QDs prepared at $180^{\circ} \mathrm{C}$ for $3 \mathrm{~min}$ and resuspended in either toluene (left) or chloroform (right). The right pair compares QDs prepared at $225^{\circ} \mathrm{C}$ for $3 \mathrm{~min}$ and resuspended in either toluene (left) or 1-octadecene (right).

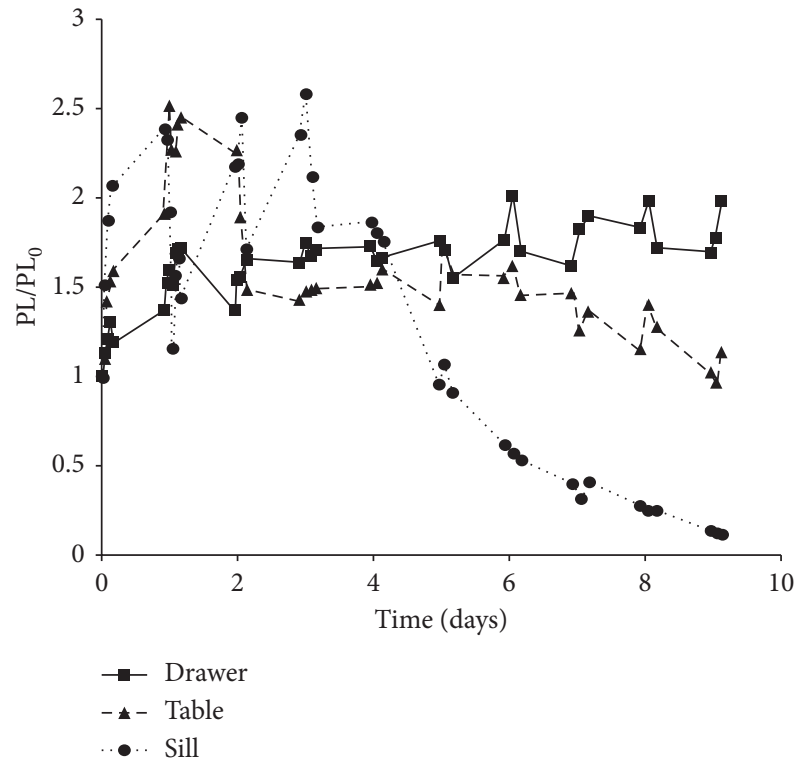

(a)

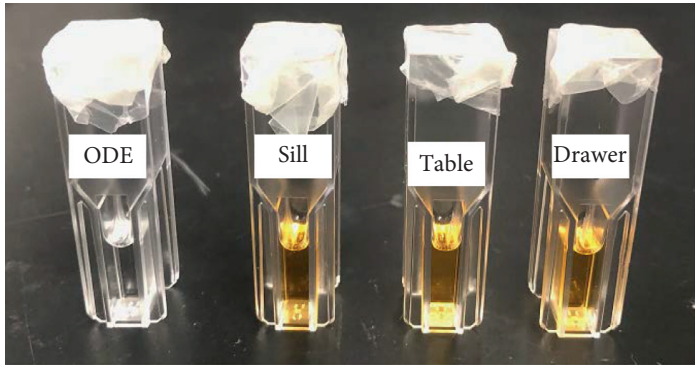

(b)

FIGURE 6: Continued. 


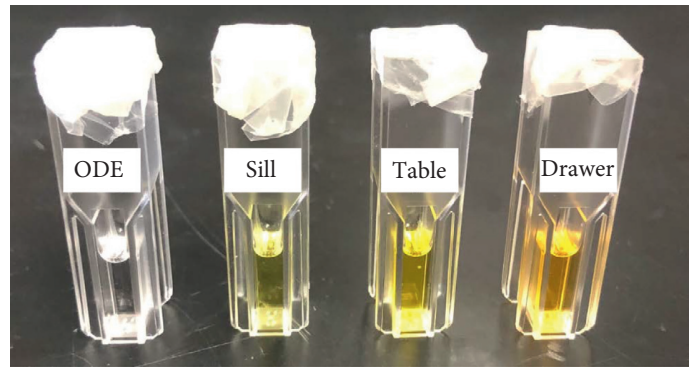

(c)

FIgURE 6: Plot of time-dependent photoluminescence (PL)/initial photoluminescence $\left(\mathrm{PL}_{0}\right)(\mathrm{a})$, image at $t=0$ days $(\mathrm{b})$, and image at $t=9$ days (c) for comparative samples of CdSe QD solutions $\left(170^{\circ} \mathrm{C} ; 2 \mathrm{~min} 30 \mathrm{~s}\right)$. QDs were either left in a closed lab drawer with limited exposure to ambient light (solid line, square shape), placed on an open laboratory tabletop with continual exposure to mostly fluorescent light (dashed line, triangle shape), or placed on an interior windowsill with frequent exposure to sunlight and fluorescent light (dotted line, circle shape) for the course of the experiment. In the pictures from left to right, the samples are neat 1-octadecene, QDs left on the windowsill, QDs left on the tabletop, and QDs stored in the drawer, respectively.

few days of continuous photoexcitation and dramatically reduces the PL intensity of QDs within 5-7 days. The difference in the rate of PL intensity decline between the windowsill sample and the tabletop sample indicates that the sun has a larger influence on photobleaching than ambient fluorescent light, though this suggestion requires further testing for confirmation. Third, the photobleaching effect is visually apparent (Figures 6(b) and 6(c)). The synthesized QDs appeared orange at the beginning of experimentation (Figure 6(b)), but by day 9 (Figure 6(c)), both the windowsill sample and the tabletop sample experienced a noticeable blue shift in color.

\section{Conclusions}

It is possible to synthesize CdSe QDs by using a microwave synthesis reactor instead of the cumbersome glassware and heating elements designed for a hot-injection method, all while using the same materials and precursor preparations of the latter. In addition to being a valuable tool in the classroom for learning about quantum confinement in materials, this simple "press-and-go" microwave technique can also be up-scaled to research and development or industry environments where access to a microwave reactor is feasible. However, there are challenges and limitations to consider with this technique such as the blue shifting of the QDs after purification, organic solvent immiscibility, or PLbroadening of freshly synthesized batches. Such oddities are not reported in most hot-injection or microwave synthesis publications, and so deserve exposure.

If this microwave technique were refined through replication and more research, these peculiarities would dissipate, leaving microwave synthesis as a cheap, quick, and energyefficient alternative to synthesizing QDs via the hot-injection technique. In addition to refinement, there is also the potential to try synthesizing other types of QDs based on hot-injection methods. For instance, one of the best types of QDs for solar cell applications is $\mathrm{PbS}$, with efficiencies reaching $12 \%$ and beyond [4]. To date, there are no readily available microwave synthesis methods for PbS; however, there are a number of hot-injection methods, all of which could be adapted to a microwave heating procedure. The research potential for microwave synthesis is immense, and with time, it could come to be the industry standard for QD synthesis and implementation.

\section{Data Availability}

The data used to support the findings of this study are available from the corresponding author upon request.

\section{Conflicts of Interest}

The authors declare that there are no conflicts of interest regarding the publication of this paper.

\section{Acknowledgments}

The authors would like to thank Travis Greene, Mark Swift, and Samantha Koutsares for their technical support, research assistance, editing assistance, and encouragement of ideas throughout this process. This work was supported by the National Science Foundation (CREST, grant number HRD 1547771; CREST, grant number HRD 1036494).

\section{References}

[1] L. Brus, "Electronic wave functions in semiconductor clusters: experiment and theory," The Journal of Physical Chemistry, vol. 90, no. 12, pp. 2555-2560, 1986.

[2] T. Kippeny, L. A. Swafford, and S. J. Rosenthal, "Semiconductor nanocrystals: a powerful visual aid for introducing the particle in a box," Journal of Chemical Education, vol. 79, no. 9, p. 1094, 2002.

[3] V. I. Klimov, Nanocrystal Quantum Dots, CRC Press, Boca Raton, FL, USA, 2nd edition, 2010.

[4] J. Xu, O. Voznyy, M. Liu et al., "2D matrix engineering for homogeneous quantum dot coupling in photovoltaic solids," Nature Nanotechnology, vol. 13, no. 6, pp. 456-462, 2018.

[5] P. V. Kamat, "Quantum dot solar cells. Semiconductor nanocrystals as light harvesters," The Journal of Physical Chemistry C, vol. 112, no. 48, pp. 18737-18753, 2008. 
[6] Y. Shirasaki, G. J. Supran, M. G. Bawendi, and V. Bulović, "Emergence of colloidal quantum-dot light-emitting technologies," Nature Photonics, vol. 7, no. 1, pp. 13-23, 2013.

[7] C. R. Kagan, E. Lifshitz, E. H. Sargent, and D. V. Talapin, "Building devices from colloidal quantum dots," Science, vol. 353, no. 6302, 2016.

[8] M. L. Landry, T. E. Morrell, T. K. Karagounis, C.-H. Hsia, and C.-Y. Wang, "Simple syntheses of CdSe quantum dots," Journal of Chemical Education, vol. 91, no. 2, pp. 274-279, 2014.

[9] C. B. Murray, D. J. Norris, and M. G. Bawendi, "Synthesis and characterization of nearly monodisperse CdE ( $\mathrm{E}=$ sulfur, selenium, tellurium) semiconductor nanocrystallites," Journal of the American Chemical Society, vol. 115, no. 19, pp. 8706-8715, 1993.

[10] M. B. Mohamed, D. Tonti, A. Al-Salman, A. Chemseddine, and M. Chergui, "Synthesis of high quality zinc blende CdSe nanocrystals," The Journal of Physical Chemistry B, vol. 109, no. 21, pp. 10533-10537, 2005.

[11] G. H. Carey, A. L. Abdelhady, Z. Ning, S. M. Thon, O. M. Bakr, and E. H. Sargent, "Colloidal quantum dot solar cells," Chemical Reviews, vol. 115, no. 23, pp. 12732-12763, 2015.

[12] K. J. Nordell, E. M. Boatman, and G. C. Lisensky, "A safer, easier, faster synthesis for CdSe quantum dot nanocrystals," Journal of Chemical Education, vol. 82, no. 11, p. 1697, 2005.

[13] M. Z. Hu and T. Zhu, "Semiconductor nanocrystal quantum dot synthesis approaches towards large-scale industrial production for energy applications," Nanoscale Research Letters, vol. 10, no. 1, pp. 1-15, 2015.

[14] O. Palchik, R. Kerner, A. Gedanken, A. M. Weiss, M. A. Slifkin, and V. Palchik, "Microwave-assisted polyol method for the preparation of CdSe "nanoballs"," Journal of Materials Chemistry, vol. 11, no. 3, pp. 874-878, 2001.

[15] J. Zhu, O. Palchik, S. Chen, and A. Gedanken, "Microwave assisted preparation of $\mathrm{CdSe}, \mathrm{PbSe}$, and $\mathrm{Cu}_{2-x} \mathrm{Se}$ nanoparticles," The Journal of Physical Chemistry B, vol. 104, no. 31, pp. 7344-7347, 2002.

[16] D. W. Ayele, H.-M. Chen, W.-N. Su et al., "Controlled synthesis of CdSe quantum dots by a microwave-enhanced process: a green approach for mass production," Chemistry- $A$ European Journal, vol. 17, no. 20, pp. 5737-5744, 2011.

[17] M. M. Moghaddam, M. Baghbanzadeh, A. Keilbach, and C. O. Kappe, "Microwave-assisted synthesis of CdSe quantum dots: can the electromagnetic field influence the formation and quality of the resulting nanocrystals?," Nanoscale, vol. 4, no. 23, pp. 7435-7442, 2012.

[18] J. A. Gerbec, D. Magana, A. Washington, and G. F. Strouse, "Microwave-enhanced reaction rates for nanoparticle synthesis," Journal of the American Chemical Society, vol. 127, no. 45, pp. 15791-15800, 2005.

[19] A. L. Washington and G. F. Strouse, "Microwave synthesis of CdSe and CdTe nanocrystals in nonabsorbing alkanes," Journal of the American Chemical Society, vol. 130, no. 28, pp. 8916-8922, 2008.

[20] J. Ziegler, A. Merkulov, M. Grabolle, U. Resch-Genger, and T. Nann, "High-quality ZnS shells for CdSe nanoparticles: rapid microwave synthesis," Langmuir, vol. 23, no. 14, pp. 7751-7759, 2007.

[21] D. A. Hines and P. V. Kamat, "Recent advances in quantum dot surface chemistry," ACS Applied Materials \& Interfaces, vol. 6, no. 5, pp. 3041-3057, 2014.

[22] Anton Paar, Monowave 300 Microwave Synthesis Reactor: Original Instruction Manual, Anton Paar, Graz, Austria, 2015.
[23] A. J. Morris-Cohen, M. D. Donakowski, K. E. Knowles, and E. A. Weiss, "The effect of a common purification procedure on the chemical composition of the surfaces of CdSe quantum dots synthesized with trioctylphosphine oxide," The Journal of Physical Chemistry C, vol. 114, no. 2, pp. 897-906, 2010.

[24] Aldrich Chemistry, Lumidot ${ }^{\mathrm{TM}}$ FAQ: Frequently Asked Questions about Using QD Nanocrystals, Aldrich Chemistry, St. Louis, MO, USA, 2019, https://www.sigmaaldrich.com/content/dam/sigmaaldrich/docs/Sigma-Aldrich/General_Information/lumidot_faqs. pdf.

[25] D. Zhou, M. Lin, Z. Chen et al., "Simple synthesis of highly luminescent water-soluble CdTe quantum dots with controllable surface functionality," Chemistry of Materials, vol. 23, no. 21, pp. 4857-4862, 2011.

[26] E. Jenrette, S. K. Pradhan, G. Rutherford, J. Flowers, D. Ha, and A. K. Pradhan, "Quantum-dot-conjugated graphene oxide as an optical tool for biosensor," Optics Express, vol. 23, no. 19, p. 25017, 2015.

[27] E. Zillner, S. Fengler, P. Niyamakom, F. Rauscher, K. Köhler, and T. Dittrich, "Role of ligand exchange at CdSe quantum dot layers for charge separation," The Journal of Physical Chemistry C, vol. 116, no. 31, pp. 16747-16754, 2012.

[28] D. B. Tice, M. T. Frederick, R. P. H. Chang, and E. A. Weiss, "Electron migration limits the rate of photobrightening in thin films of CdSe quantum dots in a dry $\mathrm{N}_{2}$ (g) atmosphere," The Journal of Physical Chemistry C, vol. 115, no. 9, pp. 3654-3662, 2011.

[29] W. G. J. H. M. van Sark, P. L. T. M. Frederix, A. A. Bol, H. C. Gerritsen, and A. Meijerink, "Blueing, bleaching, and blinking of single CdSe/ZnS quantum dots," ChemPhysChem, vol. 3, no. 10, pp. 871-879, 2002.

[30] N. Gilroy, Direct Normal Solar Resource of Virginia, National Renewable Energy Laboratory for the U.S. Department of Energy, Golden, CO, USA, 2017, https://www.nrel.gov/gis/ solar.html. 